\title{
Proper actions of lamplighter groups associated with free groups
}

\author{
Yves de Cornulier, Yves Stalder and Alain Valette*
}

November 17, 2018

\begin{abstract}
Given a finite group $H$ and a free group $\mathbf{F}_{n}$, we prove that the wreath product $H \imath \mathbf{F}_{n}$ admits a metrically proper, isometric action on a Hilbert space.
\end{abstract}

\section{Introduction}

Following [HP98, a space with walls is a pair $(X, \mathcal{W})$ where $X$ is a set and $\mathcal{W}$ is a family of partitions of $X$ into two classes, called walls, such that for any two distinct points $x, y \in X$, the number $w(x, y)$ of walls separating $x$ from $y$, called the wall distance between $x$ and $y$, is finite.

Let us define the class $\mathcal{P W}$ as the class of countable groups $G$ admitting a left-invariant structure of space with walls such that the corresponding wall distance is proper, i.e. bounded subsets are finite. The class $\mathcal{P W}$ contains for instance $\mathbf{Z}^{n}$, free groups, surface groups (see 1.2.7 in $\left[\mathrm{CCJ}^{+} 01\right]$ for this fact). More generally, it contains all groups acting combinatorially properly on some finite product of trees.

It is known (see [CCJ $\left.{ }^{+} 01\right]$, Corollary 7.4.2) that, if $G$ belongs to the class $\mathcal{P W}$, then $G$ has the Haagerup property (or is $a$-T-menable), i.e. $G$ admits an isometric action on a Hilbert space $\mathcal{H}$ that is metrically proper, that is,

$$
\lim _{g \rightarrow \infty}\left\|x_{0}-g \cdot x_{0}\right\|=+\infty
$$

for some/every $x_{0}$ in $\mathcal{H}$.

${ }^{*}$ This research was done at Centre Bernoulli (EPF Lausanne), in the framework of the semester "Limits of graphs in group theory and computer science". 
Recall that the (standard, restricted) wreath product $H$ ₹ $G$ of two groups $H$ and $G$ is the semidirect product $H^{(G)} \rtimes G$, where $G$ acts by shifting the direct sum $H^{(G)}$ of copies of $H$. Up to now, the problem of stability of the Haagerup Property under wreath products was open. Indeed, the Haagerup Property is closed under direct sums, but not under general semidirect products. However it is known to be closed under extensions with amenable quotients $\mathrm{CCJ}^{+}$01, Example 6.1.6], so that in particular $H$ 乙 $G$ is Haagerup whenever $H$ is Haagerup and $G$ is amenable.

These were however the only known examples of wreath products with the Haagerup Property. This note presents the first examples of a-T-menable wreath products $H \curlyvee G$ with $H \neq 1$ and $G$ non-amenable, including the first natural such example, namely the "lamplighter group" $(\mathbf{Z} / 2 \mathbf{Z})$ 乙 $\mathbf{F}_{2}$ over the free group $\mathbf{F}_{2}$ of rank two.

Theorem 1 Let $H$ be a finite group. If $G$ is a group in $\mathcal{P} \mathcal{W}$, then so is $H$ 乙 G. In particular, $H$ \ $G$ has the Haagerup property.

This latter statement will be generalized in a forthcoming paper, where we prove that the Haagerup Property is closed under taking wreath products. The proof of the general case relies on the same basic construction, but requires more technicalities.

Theorem 1 is proved in Section 2, while Section 3 presents some consequences on the relation between the Haagerup Property and weak amenability.

Ackowledgements: Thanks are due to I. Chatterji, C. Drutu and S. Popa for useful conversations and correspondence, and to N. Ozawa for suggesting Corollary 3. The second-named author is especially grateful to Frédéric Haglund for very fruitful discussions and hints about the construction of families of walls for wreath products.

\section{Proof of Theorem 1}

We first fix some notation. Write $\Lambda=H^{(G)}$ and $\Gamma=H \curlywedge G=\Lambda \rtimes G$. Elements of $\Gamma$ are denoted

$$
\gamma=\lambda g(\lambda \in \Lambda, g \in G)
$$

The support of $\lambda$ is

$$
\operatorname{supp}(\lambda)=\left\{g \in G: \lambda(g) \neq 1_{H}\right\}
$$


If $(X, \mathcal{W})$ is a space with walls, a half-space in $X$ is one of the two classes of some wall in $\mathcal{W}$. Suppose that $G$ belongs to the class $\mathcal{P} \mathcal{W}$, and let us denote by $\mathcal{H}$ the family 1$]$ of half-spaces in $G$. For $A \in \mathcal{H}$, we denote by $A^{c}$ the other half-space in the same wall, i.e. the complement of $A$ in $G$. For $A \in \mathcal{H}$ and $\mu: A^{c} \rightarrow H$ a function with finite support, we set

$$
E(A, \mu)=:\left\{\gamma=\lambda g \in \Gamma: g \in A,\left.\lambda\right|_{A^{c}}=\mu\right\} .
$$

We define a family of walls in $\Gamma$ as partitions $\left\{E(A, \mu), E(A, \mu)^{c}\right\}$, for $A \in \mathcal{H}$ and $\mu: A^{c} \rightarrow H$ finitely supported. We check in three steps that this equips $\Gamma$ with a structure of space with walls on which $\Gamma$ acts properly.

1st step: $\Gamma$ is a space with walls.

Let $\gamma_{1}=\lambda_{1} g_{1}$ and $\gamma_{2}=\lambda_{2} g_{2}$ be two elements of $\Gamma$. Let us show that there are finitely many $E(A, \mu)$ 's such that $\gamma_{1} \in E(A, \mu)$ and $\gamma_{2} \notin E(A, \mu)$.

Indeed $\gamma_{1} \in E(A, \mu)$ means $g_{1} \in A$ and $\left.\lambda_{1}\right|_{A^{c}}=\mu$ (so that $\mu$ is determined once $A$ is given). And $\gamma_{2} \notin E(A, \mu)$ means that either $g_{2} \notin A$, or $\left.\lambda_{2}\right|_{A^{c}} \neq \mu$; since $\mu=\left.\lambda_{1}\right|_{A^{c}}$, this can be re-written:

$$
A^{c} \cap\left(\left\{g_{2}\right\} \cup \operatorname{supp}\left(\lambda_{1}^{-1} \lambda_{2}\right)\right) \neq \emptyset .
$$

So $A$ must separate $g_{1}$ from the finite set $\left\{g_{2}\right\} \cup \operatorname{supp}\left(\lambda_{1}^{-1} \lambda_{2}\right)$. Since $G$ is a space with walls, this singles out finitely many possibilities for $a$.

2nd step: $\Gamma$ preserves the above wall structure.

This follows immediately from the formulae:

$$
\begin{gathered}
g E(A, \mu)=E(g A, g \mu) \quad(g \in G) ; \\
\lambda E(A, \mu)=E\left(a,\left.\lambda\right|_{A^{c}} \mu\right) \quad(\lambda \in \Lambda) .
\end{gathered}
$$

3rd step: $\Gamma$ acts metrically properly on its wall structure.

Let $w_{\Gamma}(\gamma)$ be the number of walls separating the unit $1_{\Gamma}$ from $\gamma \in \Gamma$. We must prove that, for every $N \in \mathbf{N}$, there are finitely many $\gamma$ 's with $w_{\Gamma}(\gamma) \leq N$.

Define analogously $w_{G}(g)$ as the number of walls separating $1_{G}$ from $g$ in $G$, and set $B_{G}(N)=\left\{g \in G: w_{G}(g) \leq N\right\}$; by our assumption $B_{G}(N)$ is a finite set.

\footnotetext{
${ }^{1}$ Although the family is not assumed injective, we will identify, to avoid subscripts in the notation, elements of the index set $\mathcal{H}$ with the corresponding subsets of $G$.
} 
Claim: If $\gamma=\lambda g$ satisfies $w_{\Gamma}(\gamma) \leq N$, then $\{g\} \cup \operatorname{supp}(\lambda) \subset B_{G}(N)$.

Theorem 1 then follows from the claim together with the fact that $H$ is a finite group.

Proof of the claim: Contraposing, suppose that there exists $g^{\prime} \in\{g\} \cup$ $\operatorname{supp}(\lambda)$ with $w_{G}\left(g^{\prime}\right)>N$. So we find $N+1$ distinct half-spaces $A_{0}, \ldots, A_{N}$ in $\mathcal{H}$ with $1_{G} \in A_{i}$ and $g^{\prime} \notin A_{i}(i=0, \ldots, N)$. Then the $E\left(A_{i}, 1_{\Lambda}\right)$ 's are $N+1$ distinct half-spaces in $\Gamma$ separating $1_{\Gamma}$ from $\gamma=\lambda g$, so $w_{\Gamma}(\gamma)>N$.

\section{Weak amenability à la Cowling-Haagerup}

Theorem 1 has interesting consequences in view of a recent result of Ozawa and Popa OP]. Recall from [CH89] that a countable group $G$ is weakly amenable if there exists a constant $L>0$ and a sequence $\left(f_{n}\right)_{n>0}$ of functions with finite support on $G$, converging pointwise to 1 , and such that $\left\|f_{n}\right\|_{c b} \leq L$ for $n>0$, where $\|f\|_{c b}$ is the Herz-Schur multiplier norm of the function $f$. The best (i.e. lowest) possible $L$ for which there exists such a sequence is the Cowling-Haagerup constant of $G$, denoted by $\Lambda(G)$. We set $\Lambda(G)=\infty$ if $G$ is not weakly amenable. Groups which are weakly amenable with constant 1 are also said to satisfy the complete metric approximation property.

In [OP, Corollary 2.11], it is proved that, if $H$ is non-trivial and $G$ is non-amenable, then $H \imath G$ does not have the complete metric approximation approximation property. Combining with Theorem 1, we get:

Corollary 2 For $H$ a non-trivial finite group, $H<\mathbf{F}_{2}$ is an a-T-menable group without the complete metric approximation property.

This disproves a conjecture of Cowling (see page 7 in $\left[\mathrm{CCJ}^{+} 01\right]$ ), stating that the class of a-T-menable groups coincides with the class of groups with the complete metric approximation property. Whether every such group is a-T-menable, is still an open question.

It was pointed out to us by N. Ozawa that from Corollary 2 one can deduce the following:

Corollary 3 Let $H$ be a non-trivial finite group. The iterated wreath product $\left(H>\mathbf{F}_{\mathbf{2}}\right)$ 乙 $\mathbf{Z}$ is a-T-menable but not weakly amenable.

Proof: It was already observed above that $G=:\left(H<\mathbf{F}_{\mathbf{2}}\right)$ $) \mathbf{Z}$ is a-T-menable; on the other hand, for $N \geq 1$ consider a subgroup $K_{N}$ of $G$ which is the 
direct sum of $N$ copies of $H \prec \mathbf{F}_{2}$. Then by Proposition 1.3.(a) and Corollary 1.5 in [CH89], we have $\Lambda(G) \geq \Lambda\left(K_{N}\right)=\Lambda\left(H \succ \mathbf{F}_{\mathbf{2}}\right)^{N}$. Since $N$ is arbitrary and $\Lambda\left(H<\mathbf{F}_{\mathbf{2}}\right)>1$ by the Ozawa-Popa result [OP], we get $\Lambda(G)=\infty$.

In view of the cubulation of spaces with walls, carried out independently in [CN05] and [Nic04], we get from Theorem 1:

Corollary 4 Let $H$ be a non-trivial finite group. The wreath product $H$ 乙 $\mathbf{F}_{2}$ admits a metrically proper, isometric action on a $C A T(0)$ cube complex, but does not have the complete metric approximation property.

In contrast, it was recently proved by Guentner and Higson [GH] that a group acting metrically properly, isometrically on a finite-dimensional $C A T(0)$ cube complex, has the complete metric approximation property.

\section{References}

[CCJ $\left.{ }^{+} 01\right]$ Pierre-Alain Cherix, Michael Cowling, Paul Jolissaint, Pierre Julg, and Alain Valette. Groups with the Haagerup property, volume 197 of Progress in Mathematics. Birkhäuser Verlag, Basel, 2001.

[CH89] Michael Cowling and Uffe Haagerup. Completely bounded multipliers of the Fourier algebra of a simple Lie group of real rank one. Invent. Math., 96(3):507-549, 1989.

[CN05] Indira Chatterji and Graham A. Niblo. From wall spaces to $\mathrm{CAT}(0)$ cube complexes. Internat. J. Algebra Comput., 15(5\&6):875-885, 2005.

[GH] Erik Guentner and Nigel Higson. Weak amenability of CAT(0) cubical groups. arXiv:math.OA/0702568 v1.

[HP98] Frédéric Haglund and Frédéric Paulin. Simplicité de groupes d'automorphismes d'espaces à courbure négative. In The Epstein birthday schrift, volume 1 of Geom. Topol. Monogr., pages 181-248 (electronic). Geom. Topol. Publ., Coventry, 1998.

[Nic04] Bogdan Nica. Cubulating spaces with walls. Algebr. Geom. Topol., 4:297-309 (electronic), 2004. 
[OP] Narutaka Ozawa and Sorin Popa. On a class of $\mathrm{II}_{1}$ factors with at most one Cartan subalgebra. arXiv:math.OA/0706.3623 v3.

\section{Authors addresses:}

Y.C. Institut de Recherche Mathématique de Rennes, Université de Rennes 1, Campus de Beaulieu, 35042 Rennes Cedex, France yves.decornulier@univ-rennes1.fr

Y.S. Laboratoire de Mathématiques, Université Blaise Pascal, Campus universitaire des Cézeaux, 63177 Aubière Cedex, France yves.stalder@math.univ-bpclermont.fr

A.V. Institut de Mathématiques, Université de Neuchâtel, Rue Émile Argand 11, CP 158, 2009 Neuchâtel, Switzerland alain.valette@unine.ch 